\title{
Grip-Pattern Verification for Smart Gun Based on Maximum-Pairwise Comparison and Mean-Template Comparison
}

\author{
Xiaoxin Shang and Raymond N. J. Veldhuis \\ Signals and Systems Group \\ Electrical Engineering \\ University of Twente \\ P.O. Box 217 \\ 7500 AE Enschede \\ The Netherlands
}

\begin{abstract}
In our biometric verification system of a smart gun, the rightful user of a gun is authenticated by grip-pattern recognition. In this work verification will be done using two types of comparison methods, respectively. One is mean-template comparison, where the matching score between a test image and a subject is computed, by comparing the test image to the mean value of training samples of this subject. The other one is maximum-pairwise comparison, where the matching score between a test image and a subject is selected as the maximum, among all the similarity scores resulting from comparison between the test image and each training sample of this subject. Experimental results show that a much lower false-acceptance rate can be obtained at the required false-rejection rate of our system using maximum-pairwise comparison, than mean-template comparison.
\end{abstract}

\section{INTRODUCTION}

We develop a prototype verification system as part of a smart gun, where the grip-pattern recognition ensures that a gun can only be fired by its rightful user. This application is intended to be used by the police, since carrying a gun in public brings considerable risks. In the US, for example, vital statistics show that about $8 \%$ of the law-enforcement officers killed in the line of duty were shot by their own weapons [1]. Particularly, this system should have a very low false-rejection rate, rendering that it is highly unlikely that a police officer could not fire his or her own gun. Currently, the official requirement in the Netherlands, for example, is that the probability of failure of a police gun be lower than $10^{-4}$. Therefore, in our work the false-rejection rate for verification must remain below this value. Under this precondition, the false-acceptance rate should be minimized.
Fig. 1 shows both the prototype of the smart gun and an example of a grip-pattern image. The sensor, used for measuring the hand-grip patterns, is a 44 by 44 piezo-resistive pressure sensor made by Tekscan Inc. One can see from Fig. 1(b) the pressure pattern of the thumb in the upper-left corner of the image, and those of the fingers in the remaining part. Note that only three fingers are present, because the index finger is near the trigger of the gun and its pressure pattern is therefore not measured. We recorded the grip patterns from a group of police officers in three sessions, with a time lapse in between [2]. The data were processed for verification, using a likelihood-ratio classifier described in [3]. The initial experimental results indicate that when the grip patterns for training and testing were recorded in the same session, the verification performance was fairly good, with an equal-error rate below $1 \%$; otherwise the performance was much worse, i.e., about $15 \%$ equal-error rate on average. Since in practice there is always be a time lapse between the data enrollment and verification, the verification performance in the across-session experiment is more relevant and therefore have to be improved.

Having analyzed the data collected in all sessions, we found that the grip patterns of a subject recorded across sessions varied greatly, even though those of this subject recorded in the same session were fairly similar [2]. There were mainly two types of acrosssession variations. First, a variation of pressure distributions occurred between the grip patterns from a subject recorded in different sessions. A second type of variation resulted from hand shift of a subject across sessions [2]. Fig. 2 shows two images recorded from one subject in two different sessions, respectively. One can see that these two images have quite differ- 


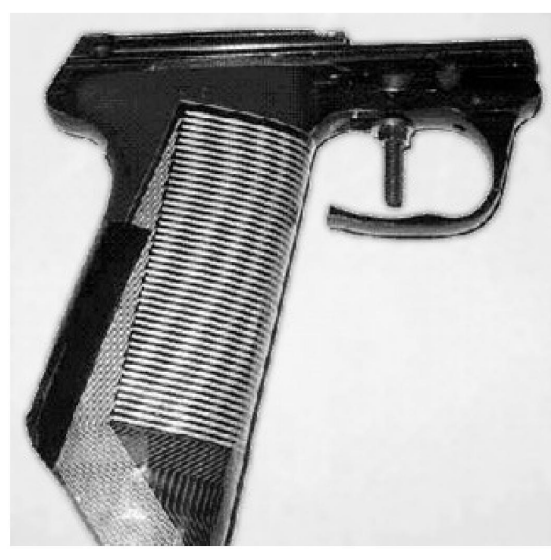

(a)

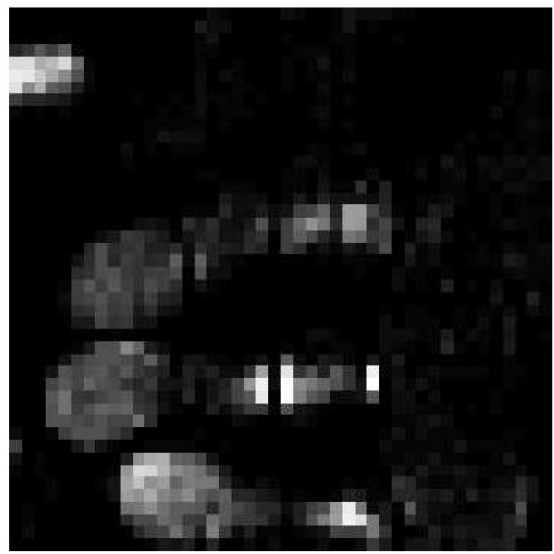

(b)

Fig. 1. (a) Prototype of the smart gun (b) An example of grippattern image

ent pressure distributions. Besides, the grip pattern in Fig. 2(b) is located higher, than that in Fig. 2(a). Further research showed that these variations were the main reason for the unsatisfactory across-session verification performance [2]. On the other hand, one can also see that the hand shape remains constant for the same subject across sessions.

Based on the characteristics of grip-pattern images described above, the verification performance may be improved by either reducing the data variations across sessions, or extracting information of the hand shapes from images. In earlier work we applied three approaches, each of which effectively improved the verification performance, respectively. First, we used template-matching registration (TMR) to reduce the across-session variation due to the hand shift [4][5].

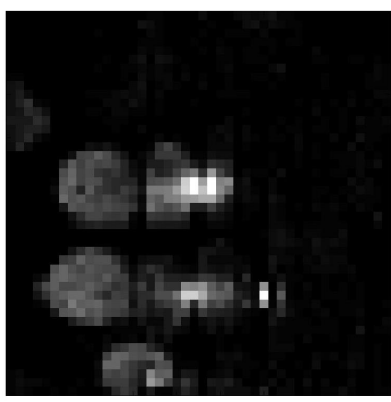

(a)

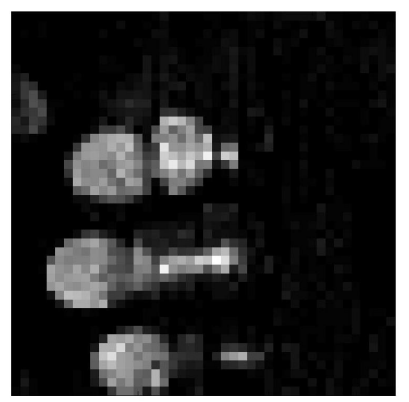

(b)
Fig. 2. Grip-pattern images of a subject in different collection sessions

This has reduced the equal-error rate to about $13 \%$ from about $15 \%$. The second technique that we applied was double-trained model (DTM), where the grip patterns from two out of three collection sessions were combined for training, and those of the remaining session were used for testing. With DTM the data variations across sessions were much better modelled during the training procedure, compared to the case where the data from only one collection session were used for training. The verification performance was greatly improved by DTM, with the equal-error rate reduced from about $15 \%$ to about $8 \%$ on average. Third, we applied an image processing approach, Local Absolute Binary Patterns (LABP), prior to classification [6]. Specifically, with respect to a certain pixel in a grip-pattern image, the LABP processing quantifies how its neighboring pixels fluctuate. This technique can not only reduce the across-session variation of the pressure distribution in the images, but also it is capable of extracting information of the hand shape from an image. It was found that the application of LABP improved the verification performance significantly, with the equal-error rate reduced from about $15 \%$ to about $9 \%$ on average. Finally, when all these three approaches were applied together the verification performance was improved greatly, yielding an average equal-error rate of $3 \%$ approximately.

Note that the verification results presented above are all given in terms of the equal-error rate, instead of the false-acceptance rate at the false-rejection rate equal to $10^{-4}$. The reason is that in earlier work we mainly focused on improving the verification performance of the system in general, and it was proved that there was no conflict between this and reducing the falseacceptance rate at the false-rejection rate equal to $10^{-4}$. 
That is, a lower equal-error rate corresponds to a lower false-acceptance rate at the required false-rejection rate, and vice versa. Also, note that the verification results presented above are all based on the mean-template comparison (MTC). That is, the matching score between a test image and a subject was obtained by comparing the test image to the mean value of training samples of this subject. In this paper we propose to use another method of comparison, namely, the maximumpairwise comparison (MPWC). With this method, a test image is compared to the training samples of a subject, one by one. Among all the similarity scores obtained, the greatest one is selected as the final matching score between the test image and this subject. In comparison with MTC, the major advantage of using MPWC is that a significantly lower false-acceptance rate for verification at the false-rejection rate equal to $10^{-4}$ can be achieved, even though no much difference in equalerror rate is produced using these two methods.

This paper presents and compares the experimental results using MPWC and MTC, respectively. The remainder of this paper is organized as follows: the verification algorithm for grip-pattern recognition will be briefly described in Section II, based on the comparison methods of MTC and MPWC respectively. Section III presents and discusses the experimental results. Finally, conclusions will be given in Section IV.

\section{VERIFICATION ALGORITHMS}

It is assumed that the grip-pattern data are Gaussian. The verification is based on a likelihood-ratio classifier. The likelihood-ratio classifier is optimal in the Neyman-Pearson sense, i.e., the false-acceptance rate is minimal at a given false-rejection rate or vice versa, if the data have a known probability density function [7][8]. The pixel values of a grip-pattern image are arranged into a (in this case $44 \times 44=1936$ dimensional) column vector $\mathrm{x}$. The feature vector $\mathrm{x}$ is normalized, i.e. $\|\mathbf{x}\|_{2}=1$, prior to classification. A measured image originates either from a genuine user, or from an impostor. The grip-pattern data of a certain subject are characterized by the local mean vector $\mu_{\mathrm{W}}$ and the local covariance matrix $\Sigma_{\mathrm{W}}$, where the subscript W denotes 'Within-class'; while the impostor data are characterized by the total mean vector $\mu_{\mathrm{T}}$ and the total covariance matrix $\Sigma_{\mathrm{T}}$, where the subscript T denotes 'Total'. The matching score of a measurement $\mathrm{x}$ with respect to this subject is derived from the loglikelihood ratio [3]. Using the comparison method of MTC, it is computed by

$$
\begin{aligned}
\mathrm{S}(\mathbf{x})= & -\left(\mathbf{x}-\mu_{\mathrm{W}}\right)^{\prime} \boldsymbol{\Sigma}_{\mathrm{W}}^{-1}\left(\mathbf{x}-\mu_{\mathrm{W}}\right) \\
& +\left(\mathbf{x}-\mu_{\mathrm{T}}\right)^{\prime} \boldsymbol{\Sigma}_{\mathrm{T}}^{-1}\left(\mathbf{x}-\mu_{\mathrm{T}}\right) .
\end{aligned}
$$

The ' denotes matrix or vector transposition. If $\mathrm{S}(\mathrm{x})$ is above a preset threshold, the measurement is accepted as being from the genuine user. Otherwise it is rejected. The threshold determines the false-rejection and falseacceptance rates for verification [3].

In practice the mean vectors and covariance matrices are unknown, and have to be estimated from a set of training data. In our case, the number of training samples from each subject should be much greater than 1936. Otherwise, the classifier would become overtrained [3]. However, we cannot make this large number of measurements, for it would be very impractical for training of the classifier.

This problem can be solved by the following steps prior to classification. First, we project all the data into a whitened PCA (Principal Component Analysis) space, such that $\Sigma_{\mathrm{T}}$ becomes an identity matrix with a lower dimensionality of $N_{\mathrm{PCA}}$. It was proved in [3], that in this new feature space, the number of modes of variations contributing to verification is not more than $N_{\text {user }}-1$, where $N_{\text {user }}$ is the number of subjects for training. And, these modes of variations have the smallest variances of the data from each individual subject. A further dimensionality reduction can then be achieved by applying a second PCA to the data, and discarding all the modes of variations except the $N_{\text {user }}-1$ ones with the smallest variances of the data from each subject. For computation in the second PCA transformation, we make a simplifying assumption that each subject shares the same within-class covariance matrix, so that it can be estimated more accurately using the data of all subjects. This last operation is in fact a dimensionality reduction by means of the LDA (Linear Discriminant Analysis).

The whole procedure of dimensionality reduction described above can be represented by a transformation matrix F. After the LDA, the total covariance matrix becomes an identity matrix, while the within-class covariance matrix becomes diagonal. The data after transformation have a dimensionality of $N_{\text {user }}-1$ [3]. As a result, (1) can be rewritten as

$$
\begin{aligned}
\mathrm{S}(\mathbf{x})= & -\left(\hat{\mathbf{x}}-\hat{\mu}_{\mathrm{W}}\right)^{\prime} \boldsymbol{\Lambda}_{\mathrm{W}}^{-1}\left(\hat{\mathbf{x}}-\hat{\mu}_{\mathrm{W}}\right) \\
& +\left(\hat{\mathbf{x}}-\hat{\mu}_{\mathrm{T}}\right)^{\prime}\left(\hat{\mathbf{x}}-\hat{\mu}_{\mathrm{T}}\right),
\end{aligned}
$$


where

$$
\begin{aligned}
\hat{\mathbf{x}} & =\mathbf{F x}, \\
\hat{\mu}_{\mathrm{W}} & =\mathbf{F} \mu_{\mathrm{W}}, \\
\hat{\mu}_{\mathrm{T}} & =\mathbf{F} \mu_{\mathrm{T}},
\end{aligned}
$$

and $\Lambda_{\mathrm{W}}$ denotes the resulting diagonal within-class covariance matrix. Therefore four entities in total need to be estimated from the training data if MTC is in use: $\mu_{\mathrm{W}}, \mu_{\mathrm{T}}, \mathrm{F}$, and $\Lambda_{\mathrm{W}}$.

If the comparison method of MPWC is applied, the data are transformed by both PCA and LDA prior to classification, in exactly the same way as in the case of MTC. We only need to change the expression of $\hat{\mu}_{\mathrm{W}}$ to:

$$
\hat{\mu}_{\mathrm{W}}=\mathbf{F m}_{i}
$$

where $\mathbf{m}_{i}, i=1, \ldots, l$ is a training sample of the subject, to whom a measurement $\mathrm{x}$ is compared.

\section{EXPERIMENTS, RESULTS AND DISCUSSION}

We recorded the grip patterns from a group of police officers in three sessions, with approximately one month and four months in between. In total, 39 subjects participated in both the first and second collection sessions, with 25 grip-pattern images recorded from each subject. In the third session, however, the data were collected from 22 subjects out of the same group of police officers, and each subject contributed 50 grippattern images.

Prior to classification, all of the three methods of TMR, DTM and LABP described in Section I were applied. The verification performance was evaluated by the false-acceptance rate at the false-rejection rate equal to $10^{-4}$, as well as the equal-error rate. Computation of both of them was based on matching scores of all the genuine users and impostors. Table I shows the experimental results obtained using MTC and MPWC, respectively. 'FARref' represents the false-acceptance rate at the false-rejection rate equal to $10^{-4}$. The 'Average' verification results were computed based on the matching scores, obtained from all cases of the combinations of training and test sessions.

Table I shows the experimental results of verification. One can see that compared to MTC, the main advantage of the application of MPWC was that the false-acceptance rate of the system has been reduced significantly on average, at the false-rejection rate equal to $10^{-4}$. It was found that this was mainly because the matching scores of those images from the genuine
TABLE I

VERIFICATION RESULTS WITH MTC AND MPWC RESPECTIVELY

\begin{tabular}{|c|c|c|c|c|c|}
\hline \multirow{2}{*}{ Train } & \multirow{2}{*}{ Test } & \multicolumn{2}{|c|}{ Equal-error rate (\%) } & \multicolumn{2}{c|}{ FARref (\%) } \\
\cline { 3 - 6 } & & MTC & MPWC & MTC & MPWC \\
\hline \hline $2+3$ & 1 & 2.0 & 2.6 & 63 & 10 \\
\hline $1+3$ & 2 & 3.6 & 3.3 & 65 & 50 \\
\hline $1+2$ & 3 & 4.9 & 4.4 & 60 & 50 \\
\hline \multicolumn{2}{|c|}{ Average } & 3.2 & 3.6 & 57 & 45 \\
\hline
\end{tabular}

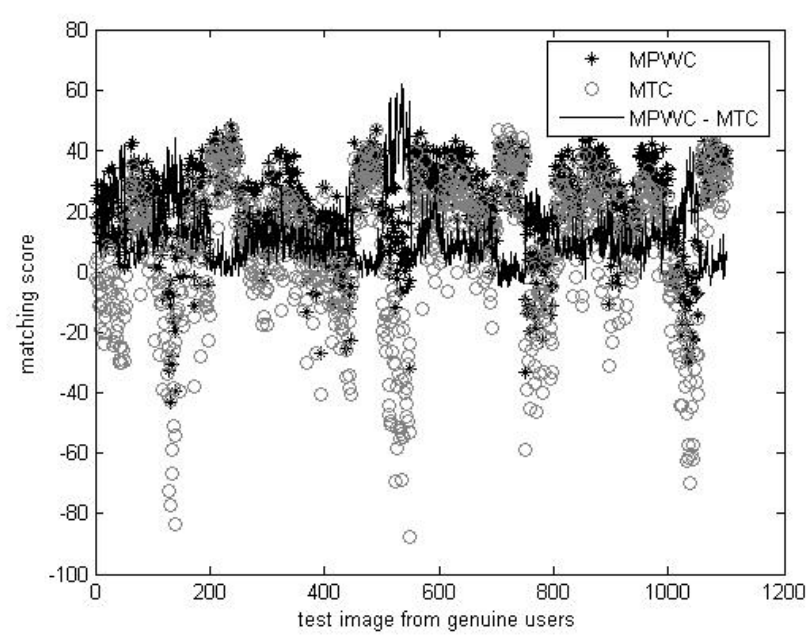

Fig. 3. Matching scores of images from genuine users with MTC and MPWC respectively

users, which were of relatively low values with MTC, increased significantly when MPWC was used instead.

Fig. 3 illustrates how the matching scores of images from the genuine users differ with the application of MPWC and MTC, respectively. In this example, the grip patterns from the first collection session are used for training and those from the third session for testing. One can see from Fig. 3 that in general the smaller matching score a test image has with MTC applied, the more it increases with MPWC. This can be explained as follows. If a test image from a genuine user has a relatively low matching score to this subject with MTC applied, it is mainly due to a large mismatch between this test image and the mean value of training samples from the subject. Thus, most likely there is a great mismatch between this test image and most of the training samples of the subject. However, as long as there exists at least one training sample, which is fairly similar to the test image, the matching score may increase significantly with MPWC applied.

The explanation given above can be demonstrated with an example, as shown in Fig. 4. Experimental result indicates that the matching score of the test image in Fig. 4(a) to the genuine user is much higher with 


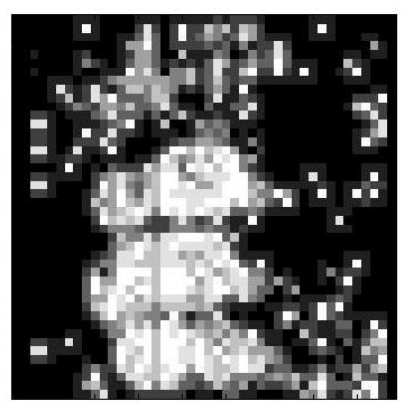

(a)

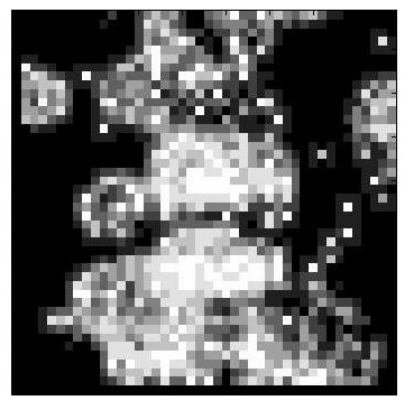

(c)

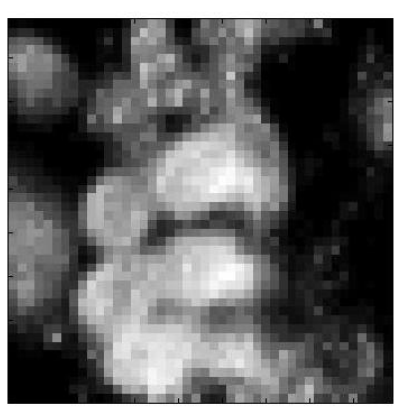

(b)

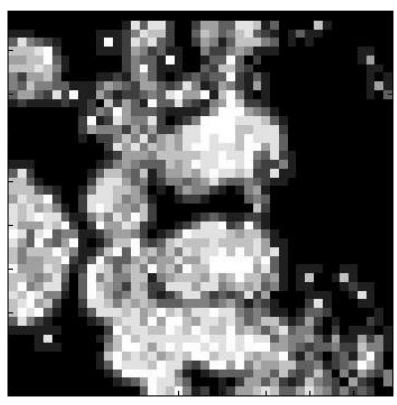

(d)
Fig. 4. (a) Test image from a genuine user (b) Mean of training samples of the genuine user (c) Training sample giving the maximum matching score (d) Training sample giving the minimum matching score

the application of MPWC, than MTC. Comparing all the four grip-pattern images, one can see that there is a big "blob" in the middle-left part of both the mean image of the training samples, and the training sample which results in the minimum matching score among all the training samples; while a "blob" does not exist in a similar location in either the test image itself, or the training sample which results in the maximum matching score. The reason that the "blob" presents in some training samples yet does not in the others, is due to the fact that while the subject holds the gun a part of the palm near the wrist touches, now and then, the sensor around the grip of the gun and exerts pressure onto it. We believe that among other factors, the absence of this "blob" has contributed to a higher matching score between the test image in Fig. 4(a) and the training sample image in Fig. 4(c).

\section{CONCLUSiONS}

The grip-pattern verification for a smart gun has been done based on the mean-template comparison and the maximum-pairwise comparison. It has been shown that a much lower false-acceptance rate for verification, at the required false-rejection rate, can be obtained using maximum-pairwise comparison than mean-template comparison. This is because it is very likely that the variations between a certain training sample of a subject and a test image from the same subject are much smaller, than the variations between the mean of training samples of this user and the test image.

\section{ACKNOWLEDGMENTS}

This research is supported by the Technology Foundation STW, applied science division of NWO, and the technology programme of the Ministry of Economic Affairs.

\section{REFERENCES}

[1] The national Uniform Crime Reporting Program. Law enforcement officers killed and assaulted. Federal Bureau of Investigation, 2001.

[2] X. Shang, R. N. J. Veldhuis, A. M. Bazen, and W. P. T. Ganzevoor. Algorithm design for grip-pattern verification in smart gun. In 16th Annual Workshop on Circuits, Systems and Signal Processing (ProRISC), 2005.

[3] R. N. J. Veldhuis, A. M. Bazen, J. A. Kauffman, and P. H. Hartel. Biometric verification based on grip-pattern recognition. In Security, Steganography, and Watermarking of Multimedia Contents, pages 634-641, 2004.

[4] A. K. Jain. Fundamentals of Digital Image Processing. Information and System Sciences. Prentice Hall, 1989.

[5] X. Shang and R. N. J. Veldhuis. Registration of hand-grip pattern in smart gun. In 17th Annual Workshop on Circuits, Systems and Signal Processing (ProRISC), 2006.

[6] X. Shang and R. N. J. Veldhuis. Local absolute binary patterns as image preprocessing for grip-pattern recognition in smart gun. In IEEE First International Conference on Biometrics. Theory, Applications and Systems (BTAS), 2007.

[7] H. L. Van Trees. Detection, Estimation, and Modulation Theory. Wiley, New York, 1968.

[8] A. M. Bazen and R. N. J. Veldhuis. Likelihood-ratio-based biometric verification. IEEE Trans. Circuits Syst. Video Techn., 14(1):86-94, 2004. 